\title{
Anticipatory attention: an event-related desynchronization approach
}

\author{
Marcel C.M. Bastiaansen ${ }^{\mathrm{a}, \mathrm{b}, *}$, Cornelis H.M. Brunia ${ }^{\mathrm{a}, \mathrm{c}}$ \\ ${ }^{\text {a } C o o p e r a t i o n ~ C e n t r e ~ T i l b u r g ~ a n d ~ E i n d h o v e n ~ U n i v e r s i t i e s, ~ T i l b u r g, ~ T h e ~ N e t h e r l a n d s ~}$ \\ ${ }^{\mathrm{b}}$ Max Planck Institute for Psycholinguistics, P.O. Box 310, 6500 AH, Nijmegen, The Netherlands \\ ${ }^{\mathrm{c}}$ Psychonomics Section, Tilburg University, Tilburg, The Netherlands
}

\begin{abstract}
This paper addresses the question of whether anticipatory attention - i.e. attention directed towards an upcoming stimulus in order to facilitate its processing - is realized at the neurophysiological level by a pre-stimulus desynchronization of the sensory cortex corresponding to the modality of the anticipated stimulus, reflecting the opening of a thalamocortical gate in the relevant sensory modality. It is argued that a technique called Event-Related Desynchronization (ERD) of rhythmic 10-Hz activity is well suited to study the thalamocortical processes that are thought to mediate anticipatory attention. In a series of experiments, ERD was computed on EEG and MEG data, recorded while subjects performed a time estimation task and were informed about the quality of their time estimation by stimuli providing Knowledge of Results (KR). The modality of the KR stimuli (auditory, visual, or somatosensory) was manipulated both within and between experiments. The results indicate to varying degrees that preceding the presentation of the KR stimuli, ERD is present over the sensory cortex, which corresponds to the modality of the KR stimulus. The general pattern of results supports the notion that a thalamocortical gating mechanism forms the neurophysiological basis of anticipatory attention. Furthermore, the results support the notion that Event-Related Potential (ERP) and ERD measures reflect fundamentally different neurophysiological processes. (c) 2001 Elsevier Science B.V. All rights reserved.
\end{abstract}

Keywords: Anticipatory attention; Thalamocortical circuits; Event-related desynchronization; Stimulus-preceding negativity; Slow potentials; Oscillations; EEG; MEG

\section{Introduction}

Most of the events that we experience everyday do not happen unexpectedly. This enables us to

\footnotetext{
* Corresponding author. Tel.: +31-24-352-15-71; fax: +3124-352-12-13.

E-mail address: marcel.bastiaansen@mpi.nl (M.C. Bastiaan-
} sen). anticipate events that can be expected to happen in the near future. Anticipatory behavior plays a ubiquitous role in everyday life. As Requin et al. (1991, p. 360) state it, 'at any moment a large part of the present activity of an organism is devoted to preparing for subsequent behavior'. Anticipatory behavior has two components, a motor component that ameliorates the processes at the output side of the organism, and a perceptual com- 
ponent that enhances the processes at the input side of the organism. The latter process, which can be termed anticipatory attention, is the focus of the present paper.

The neurophysiological model for anticipatory behavior described by Brunia (this issue), the so-called thalamocortical gating model, predicts that anticipatory attention manifests itself as a cortical activation preceding the presentation of stimuli, which is restricted to, or at least maximal in, the cortical area corresponding to the modality of the stimulus. This cortical activation would stem from an enhanced thalamocortical transfer in the relevant modality, and would serve the function of pre-setting the necessary physiological processes in the sensory cortex, in order to achieve a faster and/or more efficient processing of the upcoming sensory input.

Two EEG/MEG measures might be appropriate for revealing such cortical activation: negative slow potentials (or slow fields in case of MEG); and Event-Related Desynchronization. Negative slow potentials stem from a predominance of excitatory post-synaptic potentials at the apical dendrites of cortical pyramidal neurons, leading to a subthreshold depolarization of these cells. The concurrent increase in the readiness of cortical pyramidal neurons to fire might form the neurophysiological substrate of anticipatory attention. On the other hand, desynchronization of $10-\mathrm{Hz}$ rhythms in the EEG/MEG over the human neocortex most probably reflects a thalamically driven disruption of synchronized activity in functionally related groups of cortical neurons (cf. Lopes da Silva, this issue; Steriade et al., 1990), which can be seen as a transition of cortical idling to cortical activity. Thus, desynchronization of $10-\mathrm{Hz}$ rhythms in the EEG/MEG in sensory cortex preceding the presentation of a stimulus in the corresponding modality may be an expression of the thalamocortical gating mechanism that is proposed by Brunia and van Boxtel (this issue) to mediate anticipatory attention.

In the last two decades, quite some attention has been devoted at identifying slow negative potential shifts related to the anticipation of sensory input (cf. Böcker et al., this issue; Böcker and Van Boxtel, 1997). To this end, a paradigm has been developed in which subjects are asked to perform a time estimation task, and are subsequently informed about the quality of their time estimation by a stimulus providing Knowledge of Results (KR; Damen and Brunia, 1987). Preceding the KR stimulus, a negative slow potential can be recorded that has been termed the stimuluspreceding negativity (SPN). Note that any measure of brain activity that is a candidate for being a reflection of a purely perceptual anticipatory process as described in the thalamocortical gating model, would have to fulfil two criteria: it should be (1) independent of the type of information conveyed by the anticipated stimulus (that is, independent of whether the stimulus provides, for example, instructions on a subsequent task, or Knowledge of Results about a previously executed task); and (2) maximal over the sensory cortex corresponding to the modality of the stimulus. Subsequent research has been aimed at clarifying whether the SPN fulfils these criteria. Since detailed reviews have been presented elsewhere (e.g. Böcker et al., this issue; Böcker and Van Boxtel, 1997), we describe only the main results of these studies here. As far as the first criterion is concerned, the experimental evidence shows that the amplitude and lateralization of the SPN vary with the type of stimulus that is anticipated (cf. Böcker and Van Boxtel, 1997). With respect to modality differences in SPN topography, up to now only one study has addressed the question (Böcker et al., 1994). In this study, there was a striking similarity in scalp topography of the SPN for the two modalities: both had a frontotemporal maximum. Thus, the Böcker et al. study does not support the hypothesis that the SPN is maximal, or has a clearly distinguishable component, over the sensory cortex corresponding to the modality of the anticipated stimulus. However, recently Brunia and van Boxtel (unpublished data) demonstrated a small but significant difference in SPN scalp topography preceding auditory and visual KR stimuli, that went in the expected direction. Thus, although a small part of the SPN may be specific to the modality of the stimulus, the results do not support the hypothe- 
sis that the SPN is maximal over the sensory cortex corresponding to the modality of the anticipated stimulus.

It may be argued that analyzing the induced reactivity [e.g. by means of computing the EventRelated Desynchronization (ERD) and Event-Related Synchronization (ERS); cf. Pfurtscheller and Lopes da Silva, 1999a] of $10-\mathrm{Hz}$ rhythms could possibly be a more fruitful approach in studying whether thalamocortical gating mechanisms are at the basis of anticipatory attention rather than studying slow potentials. Considering the processes that are thought to underlie the genesis of $10-\mathrm{Hz}$ rhythms (cf. Lopes da Silva, this issue; Steriade et al., 1990), it seems reasonable to assume that ERD/ERS in the $10-\mathrm{Hz}$ range can provide us with a window on the processes that operate in thalamocortical circuits. More specifically, ERD has been associated with an enhanced thalamocortical transfer (i.e. the opening of a sensory 'gate' to the cortex), while ERS has been associated with a disruption of such transfer (Lopes da Silva, 1991; see also Guillery et al., 1998). Therefore, it may be argued that if results obtained with ERD/ERS analyses fulfil the above mentioned two criteria for being a reflection of a purely perceptual anticipatory process, one would have a valuable empirical argument in favor of the thalamocortical gating model for anticipatory attention. Although mainly the SPN does not fulfil these criteria, there are good reasons to expect differences between ERP and ERD analyses in general.

One can distinguish between two types of event-related EEG/MEG activity: induced activity and evoked activity (e.g. Bullock, 1992; Pfurtscheller and Lopes da Silva, 1999b). This distinction is most easily seen when one considers rhythmic activity. An EEG rhythm (say, the occipital alpha rhythm) may be modulated by an event (say, opening of the eyes, which leads to an amplitude enhancement), while this event does not directly drive the amplitude enhancement (the eyes, or for that matter the eyelid movements, do not produce the electrical activity which is measured over occipital leads as the alpha rhythm). Thus, induced rhythms may be defined as 'oscillations caused or modulated by stimuli or state changes that do not directly drive successive cycles of the rhythm' (Bullock, 1992, p. 1). An instance of an evoked rhythm would be the steady-state response (SSR) evoked by $40-\mathrm{Hz}$ stimulation (e.g. Rockstroh et al., 1996), in which each new stimulus directly drives each successive cycle of the SSR rhythm.

While evoked activity is by definition both timeand phase-locked to an event, induced activity is time-locked, but not necessarily phase-locked. For the present purpose, the important implication is that ERD/ERS analyses contain information (non-phase-locked, or induced EEG or MEG activity) that is lost when one computes a signal average as is done in the computation of ERPs, which leaves only the phase-locked (or evoked) part of the signal in the resulting average. From a signal analytic point of view, it is very well possible that relevant information is contained in the non-phase-locked part of the EEG/MEG. This means that a possible modality specificity of anticipatory cortical activation could have been overlooked because the analyses carried out so far on negative slow potentials have focused only on the phase-locked part of the EEG/MEG signal. ERD, with its focus on non-phase-locked activity, can be used to investigate this possibility.

The existence of modality-specific $10-\mathrm{Hz}$ rhythms, alpha, mu and tau (see Bastiaansen et al., 1999a for a discussion of these rhythms), that react differentially to stimulation in one of the three main sensory modalities (visual, somatomotor and auditory, respectively) is promising, since their very existence opens the possibility that they independently may show a local desynchronization when a stimulus in the corresponding sensory modality is anticipated.

In sum, there are neurophysiological, signalanalytic and empirical arguments for the hypothesis that ERD might be a useful tool to investigate whether anticipatory attention for an upcoming stimulus, in one of the three main sensory modalities, is mediated by an enhanced thalamocortical transfer of sensory information to the cortical areas corresponding to the modality of the anticipated stimulus. Remarkably, however, although two studies have incidentally addressed the ERD related to anticipatory attention (Klimesch et al., 
1992, 1998), systematic research into the relationship between ERD and anticipatory attention, in which stimulus modality is carefully manipulated, has not been performed. The present paper describes a series of experiments that have been performed recently in order to fill this gap.

\section{General methodology}

\subsection{Experimental paradigm}

In all the experiments described in the present paper, anticipatory attention was induced in subjects by means of a time estimation task. In this task, subjects are asked to press a button $4 \mathrm{~s}$ after the presentation of an auditory warning signal. Two seconds after the button press they are informed about the quality of their time estimation by a stimulus providing Knowledge of Results (KR) in the following way: The KR stimulus (a minus sign, a horizontal bar or a plus sign) indicates whether the subject's response was too early, correctly timed, or too late, respectively. This paradigm (schematically depicted in Fig. 1) was chosen because it has been extensively used, and has generally yielded a relatively large amplitude anticipatory slow potential (i.e. the pre-KR SPN, see Böcker et al., this issue). In contrast to other types of SPN, the neuroanatomical substrates of the pre-KR SPN are better understood than those of the other SPN variants, since the processes underlying the generation of the pre-KR SPN have been studied with source localization (Böcker et al., 1994) and PET imaging techniques (Brunia et al., 2000). This facilitates comparisons between this slow potential and the hypothesized anticipatory ERD.

The modality of the KR stimulus was varied over experiments; visual, auditory and somatosensory stimuli have been used. Since one may expect that the anticipatory process is most prominent immediately preceding the presentation of the KR stimulus, the general approach has been to assess the scalp topography of the ERD in this time interval, and to establish whether this scalp topography shows systematic differences preceding stimuli of different modalities. The general

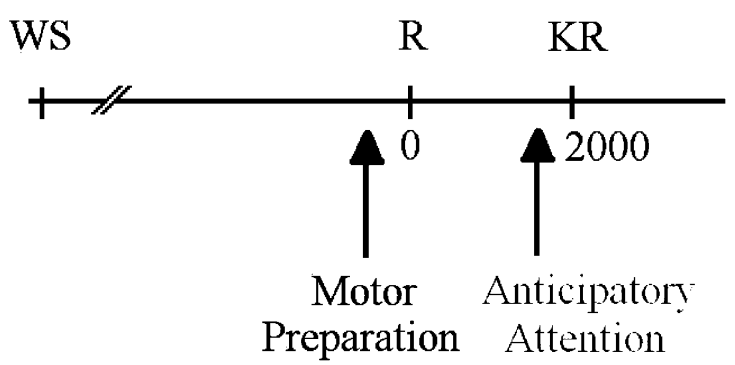

Fig. 1. The experimental paradigm used in all four experiments. WS: auditory warning signal; R: response; KR: Knowledge of Results stimulus. The process of interest, anticipatory attention, is assumed to be most active immediately prior to the presentation of the KR.

hypothesis, based on predictions of the thalamocortical gating model (Brunia, this issue; Brunia, 1999), was that ERD would be maximal over the sensory cortex corresponding to the modality of the anticipated stimulus.

\subsection{ERD computation}

The classical method used for ERD computation involves the following steps (cf. Pfurtscheller, 1999, for details): (1) applying a bandpass filter to single EEG epochs that are timelocked to an internal or external - event, leaving intact the frequency band of interest (e.g. 8-12 Hz); (2) squaring the amplitudes of the filtered EEG epochs, in order to obtain an estimate of the power in that particular frequency band; (3) integrating the power over short time intervals (e.g. $250 \mathrm{~ms}$ ) in order to obtain a more reliable estimate of the power; (4) averaging the power in each time interval over epochs; (5) expressing the power in each time interval as a percentage of the power in a reference interval or baseline. This baseline was computed, e.g. in 1-s intervals prior to each epoch, in which no task, stimulus, or response was present. The resulting measure is the average power increase (Event-Related Synchronization, ERS) or decrease (Event-Related Desynchronization, ERD) in a particular frequency band, in a particular time interval, relative to the level of the power in the baseline. This procedure can be repeated for each channel, and topographic maps can be constructed of the power 
increase or decrease. Note that when topographic mapping is desirable, it is common practice to compute the second spatial derivative, or surface Laplacian, of the EEG prior to ERD computation. The reason for this is twofold: first, it eliminates ERD effects at the reference electrodes, which can strongly influence the topography of the ERD (cf. Pfurtscheller, 1991); second, this procedure increases the spatial resolution of ERD mapping, since an inherent property of the surface Laplacian is that it acts as a spatial highpass filter. Because MEG is inherently reference-free, and has a higher spatial resolution than EEG because it is less smeared out by the low conductance of the skull, this step is omitted when ERD is computed on MEG data. A procedure for enhancing the spatial resolution of ERD computed on MEG data has been described by Bastiaansen and Knösche (2000).

It is generally agreed upon that, within the $10-\mathrm{Hz}$ frequency range, if an ERS is found at a certain area of the scalp, the underlying cortical area is inactive, while ERD corresponds to cortical activity (e.g. Pfurtscheller and Lopes da Silva, 1999b).

\section{Experiment 1: pre-stimulus ERD in the visual and auditory modalities}

\subsection{Methods}

Experiment 1 (described in detail in Bastiaansen et al., 1999b) consisted of three conditions: voluntary movement; time estimation followed by an auditory KR; and time estimation followed by a visual KR. Ten subjects participated in the experiment. In the voluntary movement task, subjects were instructed to squeeze a response manipulandum at a slow, self-paced rate (6-10 times per min). The time estimation task has been described above. Each condition was repeated twice, once with a right-hand response and once with a left-hand response. A total of 100 trials were collected in each condition for each hand.

The EEG was recorded from 23 electrode positions. Linked mastoids served as reference. EEG epochs from 3000-ms pre-movement to $3500-\mathrm{ms}$ post-movement were digitized at $128 \mathrm{~Hz}$, and amplified with a $30-\mathrm{s}$ time constant and a $30-\mathrm{Hz}$ lowpass filter. An off-line EOG correction was performed, and trials containing residual EOG, or other artifacts, were discarded from further analysis.

In order to obtain reference-free data, the recorded potentials were transformed to scalp current density (SCD) fields by estimating a spherical spline function. ${ }^{1}$ Epochs were defined from -3000 to $3500 \mathrm{~ms}$ around the response trigger, and ERD was computed along the procedure outlined above, in five different frequency bands: $8-10 \mathrm{~Hz}, 10-12 \mathrm{~Hz}, 12-16 \mathrm{~Hz}, 16-20 \mathrm{~Hz}$ and $20-25 \mathrm{~Hz}$ (cf. Bastiaansen et al., 1999b, for further details).

\subsection{Results}

The main results of the experiment are presented in Fig. 2. The pre-movement ERD and the RP both show a contralateral dominance. However, the pre-movement ERD initially starts at the contralateral side, after which a somewhat weaker ipsilateral component emerges. The RP on the contrary starts bilaterally symmetrical, and lateralizes to the contralateral side in the last 500 ms before movement onset. So far, the results are a replication of what is usually described in the literature.

Interestingly, the SPN does not show any differences in scalp distribution as a function of stimulus modality, while the pre-stimulus ERD does show clear modality specificity: preceding auditory KR stimuli there is no appreciable ERD, whereas preceding visual KR stimuli there is an occipitally maximal ERD. This finding was confirmed by the statistical analyses. It should be noted that in order to avoid a confounding of post-movement processes with pre-stimulus

\footnotetext{
${ }^{1}$ See Perrin et al., 1987, 1989. This method has the advantage of yielding more reliable estimates of the SCD at electrode positions that are at the border of the electrode montage, so it is preferable to computing a local average reference (Hjorth, 1975), as is mostly done in ERD computation (e.g. Pfurtscheller, 1991).
} 


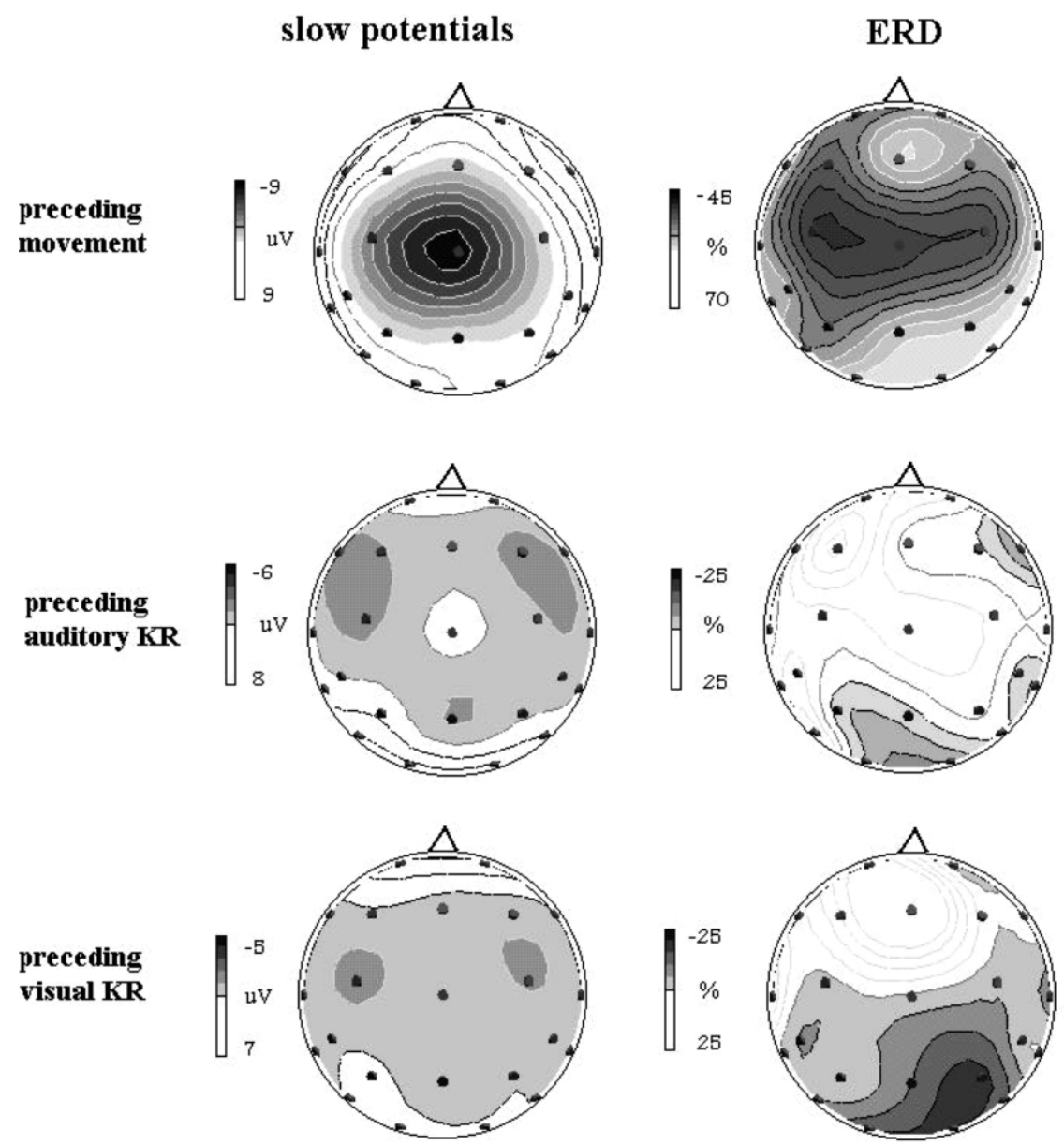

Fig. 2. Grand average $(N=10)$ slow potentials and ERD maps $(10-12 \mathrm{~Hz})$ obtained from EEG data, in the $250-\mathrm{ms}$ interval preceding response execution (top row) and KR presentation (bottom rows). Both the pre-stimulus slow potentials and the pre-stimulus ERD are based on the subtraction of activity recorded in the time estimation tasks from that recorded in the voluntary movement task, thus removing post-movement processes from the pre-stimulus processes. For the slow potentials, surface negativity is depicted as black, positivity as white. For the ERD maps, black denotes power decrease (ERD) and white power increase (ERS). Scaling as indicated. Spacing of contour lines is $1 \mu \mathrm{V}$ for the slow potential maps, and 5\% power change for the ERD maps. Note: (i) that the scalp distribution between the pre-movement potentials (readiness potential, RP) and ERD is slightly different, with the RP being maximal at the vertex and the pre-movement ERD being maximal over the contralateral hand motor area; and (ii) that preceding stimuli of different modalities the scalp distribution of the slow potentials is similar but the ERD scalp distribution shows clear differences between modalities.

processes, we subtracted the percentages ERD in the voluntary movement condition from the percentages ERD in both time estimation tasks, for the entire sampling epoch (i.e. from $3000 \mathrm{~ms}$ pre-movement to $3500 \mathrm{~ms}$ post-movement). This subtraction effectively removed all ERD/ERS effects that were related to response execution (this was confirmed by the statistical analyses on the data before and after subtraction). Therefore, the residual ERD prior to stimulus presentation can only be attributed to non-motor processes.

\subsection{Interim discussion}

Since the ERD preceding visual stimuli is maximal over the occipital cortex, it seems reasonable 
(cf. the two criteria for a measure reflecting anticipatory attention formulated in the introduction) to interpret this effect as being a reflection of an enhanced transfer in the visual thalamocortical channels. In terms of the thalamocortical gating model, it would be an opening of the visual gate, indicating anticipatory attention to the visual $\mathrm{KR}$ stimulus. Thus, although we clearly found a topographic dissociation between the two modalities (i.e. occipital ERD preceding the visual KR, and no appreciable ERD preceding the auditory KR), we did not find evidence for anticipatory attention in the auditory modality.

Niedermeyer $(1990,1991)$ reported the existence of rhythmic activity originating in the temporal lobe. This $10-\mathrm{Hz}$ rhythm could be measured by means of epidural and intracortical EEG recordings, but could not be picked up by simultaneously recorded scalp EEG. This indicates that normal, scalp recorded EEG is not well suited for measuring this type of rhythmic activity. Niedermeyer termed it the 'third rhythm', and showed that it could be functionally differentiated from alpha and mu activity on the basis of the observation that it neither displayed any reactivity to opening or closing of the eyes, nor to activity of the somato-motor system. On the other hand, Tiihonen et al. (1991) and Lehtelä et al. (1997) reported the existence of a magnetoencephalographic (MEG) rhythm originating from the temporal lobe, which was termed the tau rhythm. They demonstrated that the tau rhythm was, in most of the subjects, clearly attenuated following auditory stimulation (this effect was stronger over the right than the left hemisphere). The reactivity to auditory stimulation, together with the fact that source analyses demonstrated that the sources of the tau rhythm were located very close to the sources of the auditory evoked responses, namely in the supratemporal plane, strongly suggests that the tau rhythm is an intrinsic rhythm of the auditory cortex.

Together, these studies demonstrate that over the auditory cortex a $10-\mathrm{Hz}$ rhythm can be measured with MEG, but not with scalp recorded EEG. This observation might explain why we didn't find an ERD over the temporal cortex preceding auditory KR stimuli in our previous
EEG study (Bastiaansen et al., 1999b): if we cannot pick up rhythmic activity from the auditory cortex with scalp EEG, then we surely cannot demonstrate a desynchronization of this rhythmic activity. Experiment 2 was aimed at verifying this possibility.

\section{Experiment 2: pre-stimulus ERD: a comparative EEG / MEG study}

\subsection{Methods}

In experiment 2 (described in detail in Bastiaansen et al., 2001a), five subjects performed the time estimation task in two separate sessions, which were at least 1 week apart. Auditory and visual KR stimuli were used in different conditions. In the first session, EEG was recorded from 29 electrodes; in the second session, MEG was recorded from 64 axial gradiometers. For the MEG recordings, we used a whole-cortex MEG system (CTF Systems Inc., Vancouver, Canada), in which the sensors are uniformly distributed on the helmet surface with mean spacing of $4.5 \mathrm{~cm}$. For both EEG and MEG, epochs from $3000 \mathrm{~ms}$ pre-movement to $3000 \mathrm{~ms}$ post-movement were digitized at $256 \mathrm{~Hz}$. Filter settings were from DC to $40 \mathrm{~Hz}$. Trials that contained eye movement or other artifacts were discarded from further analysis. The ERD was computed in the 8-10 and the 10-12 Hz frequency band.

\subsection{Results}

The results displayed a considerable amount of individual variation in ERD topography in the last $250-\mathrm{ms}$ interval preceding the presentation of the KR stimuli. Therefore, all the analyses were performed at the individual level. In Fig. 3, the main results of this experiment are summarized. The ERD computed on EEG data revealed that all the subjects studied display an ERD with an occipital maximum preceding the visual KR stimulus. It should be noted that, for subjects MF and RP, the ERD preceding the visual KR stimulus is rather widespread. Preceding auditory KR stimuli, none of the subjects displayed an appre- 

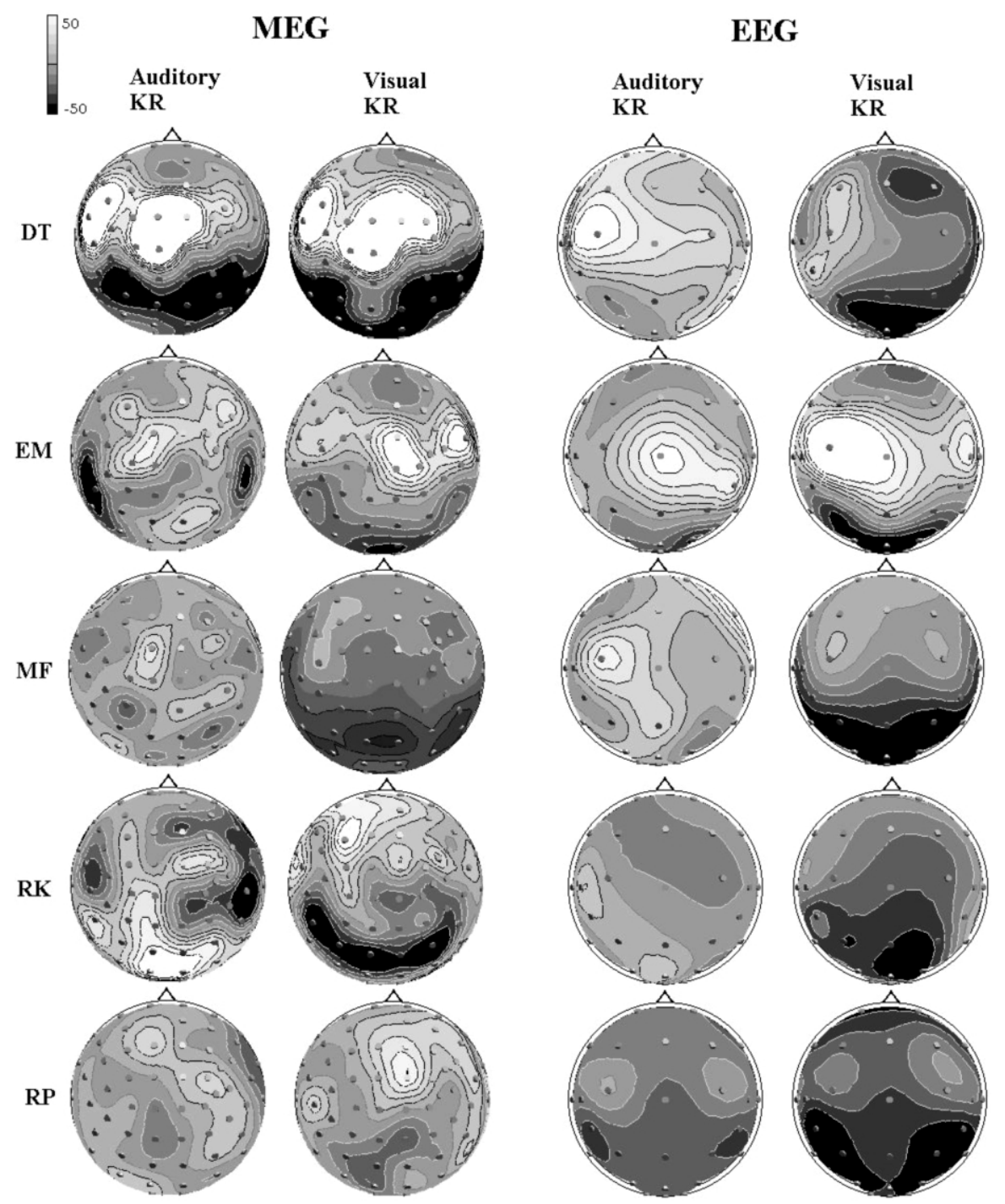

Fig. 3. Subject averages of the ERD in the $8-10-\mathrm{Hz}$ frequency band, in the last $250-\mathrm{ms}$ interval preceding the presentation of the KR stimulus. The $8-10-\mathrm{Hz}$ band is displayed because the effects were slightly stronger in this band. There were, however, no qualitative differences between the 8-10 and 10-12-hz bands. Left-hand panel: ERD on MEG data. Right-hand panel: ERD on EEG data. ERD, or power decrease, is depicted in dark gray surrounded by white contour lines, while ERS, or power increase, is depicted in light gray, surrounded by black contour lines. The gray contour lines correspond to zero power change. Scaling as indicated in the figure. Spacing of contour lines is $5 \%$ power change.

ciable ERD (see Fig. 3, right hand columns). In essence, the ERD results on the present EEG data are a confirmation of the results of our previous study (Bastiaansen et al., 1999b).

For the MEG data the picture was more complex. Therefore, we will discuss the results separately for each subject (see Fig. 3, left hand columns). Two of the five subjects (subjects EM and RK) showed a modality-dependent ERD scalp distribution in the MEG. Preceding auditory KR stimuli these subjects displayed a clear, focal and highly significant ERD over the left and right temporal areas. It should be noted that these effects were not present in the ERD computed on 
the EEG data for these two subjects. Preceding visual KR stimuli, these subjects displayed an ERD over occipital sensors, while no ERD was present over temporal sites.

One subject (subject DT) showed an occipitally maximal ERD, irrespective of KR modality, response side and frequency band. Finally, subjects MF and RP showed a significant ERD preceding the visual KR stimulus (although for subject RP this effect only borders on significance in the 8-10 $\mathrm{Hz}$ frequency band), but no ERD preceding the auditory KR stimulus.

In order to investigate the possibility that individual differences in the ERD on MEG data, over the left and right temporal sensors preceding auditory KR stimuli, were caused by between-subject differences in the level of power during baseline intervals (that is, in the presence of a tau rhythm), we computed the absolute power for each frequency band (averaged over trials, conditions and response sides, and over left and right sensors) during all the baseline intervals, for each subject separately. Means and $95 \%$ confidence intervals ( $\mathrm{M} \pm 2$ S.D.) are depicted in Fig. 4, and clearly indicate that the two subjects that display a temporal ERD preceding auditory KR stimuli (subjects EM and RK) have significantly higher levels of absolute baseline power than the subjects in which this effect is not present.

\subsection{Interim discussion}

The results of the ERD computed on the EEG data are a confirmation of the results from experiment 1. For the MEG, we also found an occipital ERD preceding visual stimuli in all of the subjects studied. There was, however, a large interindividual variability in the ERD preceding auditory stimuli: we found a clear ERD over temporal MEG sensors during anticipatory attention for an auditory stimulus only in two out of the five subjects. The main question is then, why did we find evidence for anticipatory attention for the auditory stimuli in only two out of five subjects? To (at least partially) answer this question we have to take into consideration that in these two subjects we found a larger level of absolute power over the temporal cortex. An explanation then might be that only these two subjects had a welldeveloped tau rhythm. The - relative - absence of a tau rhythm in the other three subjects might then explain the lack of desychronization in these subjects over the temporal cortex preceding auditory KR stimuli. Such a tentative explanation finds support in the results of Lehtelä et al. (1997), where (the reactivity of) the tau rhythm could not be demonstrated for all subjects, although the ratio of eight out of nine was much higher in the latter study.

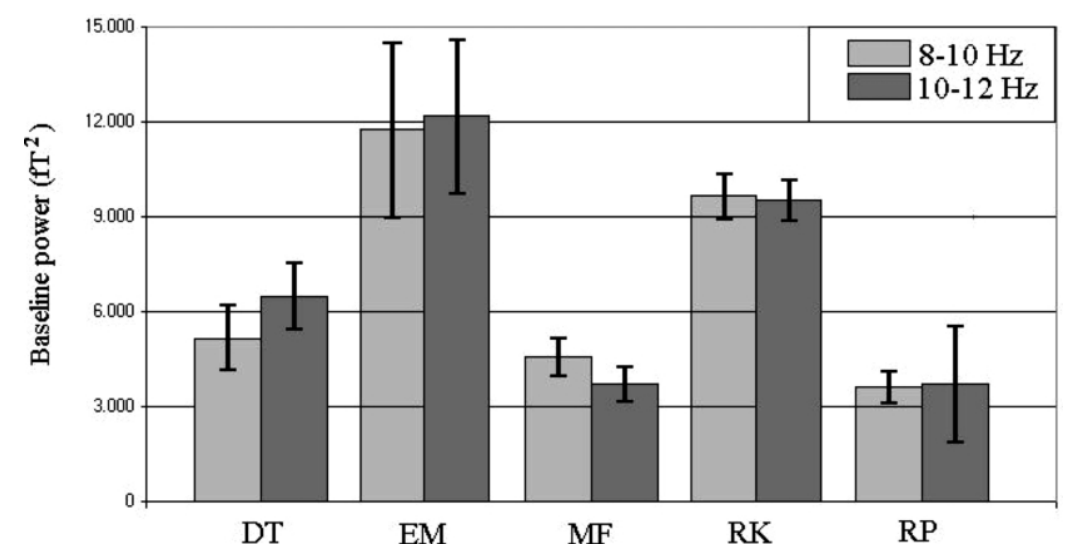

Fig. 4. Means and $95 \%$ confidence intervals (mean $\pm 2 \times$ the S.D.) of absolute power of the MEG averaged over temporal sensors during the baseline interval, for the $8-10$ and $10-12-\mathrm{Hz}$ frequency bands separately. For each subject, means and confidence intervals are computed over conditions, response sides, and averaged over left and right temporal ROIs. Note that the baseline power for subjects EM and RK is significantly larger than for the other subjects. 
Thus, it may be concluded that the data provide a weak support for the notion that anticipatory attention in the auditory modality can be detected by studying the patterns of synchronization and desynchronization of $10-\mathrm{Hz}$ rhythmical activity of the MEG. Anticipatory attention in the visual modality on the other hand can be demonstrated both with EEG and with MEG. The combined results of this and the previous study are for a large part in accordance with the predictions of the neurophysiological model of anticipatory attention that has been described elsewhere (Brunia and van Boxtel, this issue).

ERD has proved to be a very sensitive measure of cortical activation in the study of movementrelated brain activity, in the sense that the magnitude and scalp topography of the movementrelated ERD consistently vary with movement parameters (see e.g. Pfurtscheller and Lopes da Silva, 1999b, for a review). Along the same lines, if the magnitude and/or scalp topography of the pre-stimulus ERD can be influenced by varying stimulus parameters, the interpretation of this phenomenon in terms of anticipatory attention would gain strength. Therefore, an experiment was conducted in which auditory or visual KR stimuli could be either intact or perceptually degraded. If the pre-stimulus ERD reported in the first two experiments is a true index of perceptual anticipatory attention, one would expect the increase in perceptual anticipatory attention called for by stimulus degradation to be reflected in an increase in this index.

\section{Experiment 3: effects of stimulus degradation on the pre-stimulus ERD}

\subsection{Methods}

In this experiment (described in detail in Bastiaansen et al., 2001b) 12 right-handed subjects, one man and 11 women, aged 18-29 $(\mathrm{M}=21$, S.D. $=3$ ) performed a time estimation task while the EEG was measured from 27 electrodes. ERD was computed in the $8-10 \mathrm{~Hz}$ and the $10-12 \mathrm{~Hz}$ frequency bands. There were five conditions in the experiment: A voluntary movement (VM) task preceded the time estimation tasks, which were: KR auditory intact; KR auditory degraded; KR visual intact; and $\mathrm{KR}$ visual degraded.

Visual KR stimuli (duration $150 \mathrm{~ms}$ ) were presented on a 15 -inch computer monitor located 1.2 $\mathrm{m}$ in front of the subject. In the intact KR condition, the KR appeared as a white figure on a black background. In the degraded KR condition, random patterns of small squares were overlaid on the stimulus. Within each square the colors of fore- and background were inverted. A total of 20 different random square patterns was used. Auditory $\mathrm{KR}$ stimuli [70 $\mathrm{dB}(\mathrm{A}), 150 \mathrm{~ms}$ ] were presented through a loudspeaker located $1.2 \mathrm{~m}$ in front of the subject, just underneath the computer monitor. In the intact KR condition, the KR stimuli were pure tones with a frequency of 2000, 1000 or $500 \mathrm{~Hz}$, corresponding to estimated intervals that were too short, correct, or too long, respectively. In the degraded KR condition, white noise was added to the tones, but the overall output amplitude was kept constant at $70 \mathrm{~dB}(\mathrm{~A})$. If KR stimuli are more difficult to perceive as a result of stimulus degradation, but can always be accurately identified, this would be an increase in perceptual difficulty. However, if the level of degradation is such that subjects cannot in all cases reliably identify the KR stimulus, the function of the KR stimuli in guiding the time estimation on the subsequent trial will diminish, leading to an increase in task difficulty. Therefore, in a behavioral pilot study in which the KR stimuli were to be classified in three categories, for each modality a level of stimulus degradation was determined in which: (1) the RT needed for classification was larger for degraded than for intact stimuli; but (2) the percentage of correct classifications was identical to that of the intact stimuli. From the behavioral data it will be possible to infer whether stimulus degradation resulted in an increase in perceptual difficulty (in that case, the quality of time estimation is not affected by degradation) or in task difficulty (time estimation deteriorates with degradation).

\subsection{Results}

The behavioral data indicated that despite the 
efforts in selecting an appropriate level of stimulus degradation, the time estimation task was more difficult to perform when auditory degraded stimuli were used, than when the other KR stimuli were used. Furthermore, it should be noted that the SPN was largest in the auditory degraded condition. The coincidence of larger task difficulty and higher SPN amplitudes further supports the notion that the pre-KR SPN reflects the anticipation of the affective-motivational properties of the KR stimulus. This effect was not present in the pre-stimulus ERD.

The general pattern of results for the prestimulus ERD is presented in Fig. 5. Three aspects of these results are noteworthy. First, there is a strong post-movement ERS at central electrodes in the pre-stimulus interval. This is also present in the voluntary movement task, but in the latter task it is of a much smaller magnitude. Therefore, a subtraction of the VM condition from the KR conditions, as was done in experiment 1 in order to remove the post-movement ERS from the pre-stimulus ERD, could not be performed with the present data. Second, the findings of experiments 1 and 2 are replicated with the intact KR conditions (upper row in Fig.
5), in that the ERD preceding the presentation of the KR stimuli is dependent upon stimulus modality: preceding auditory stimuli, no ERD is present, whereas preceding visual stimuli, an occipital ERD is present. Third, and most importantly, preceding visual KR stimuli, the ERD is larger preceding intact, than preceding degraded stimuli, contrary to expectation.

\subsection{Interim discussion}

The results again show a clear dissociation between the ERP analyses (i.e. the pre-KR SPN) and the ERD analyses, thus supporting the notion that the different analyses reveal different types of event-related EEG/MEG responses. In general terms, whereas the pre-KR SPN varies as a function of task difficulty in the present experiment, the pre-stimulus ERD differs mostly with stimulus modality. However, stimulus degradation did not have the expected effect on the magnitude of pre-stimulus ERD: if the pre-stimulus ERD is a correlate of a perceptually based anticipatory attention process, we would expect it to be larger preceding degraded stimuli, than preceding intact stimuli. Although the finding of the present

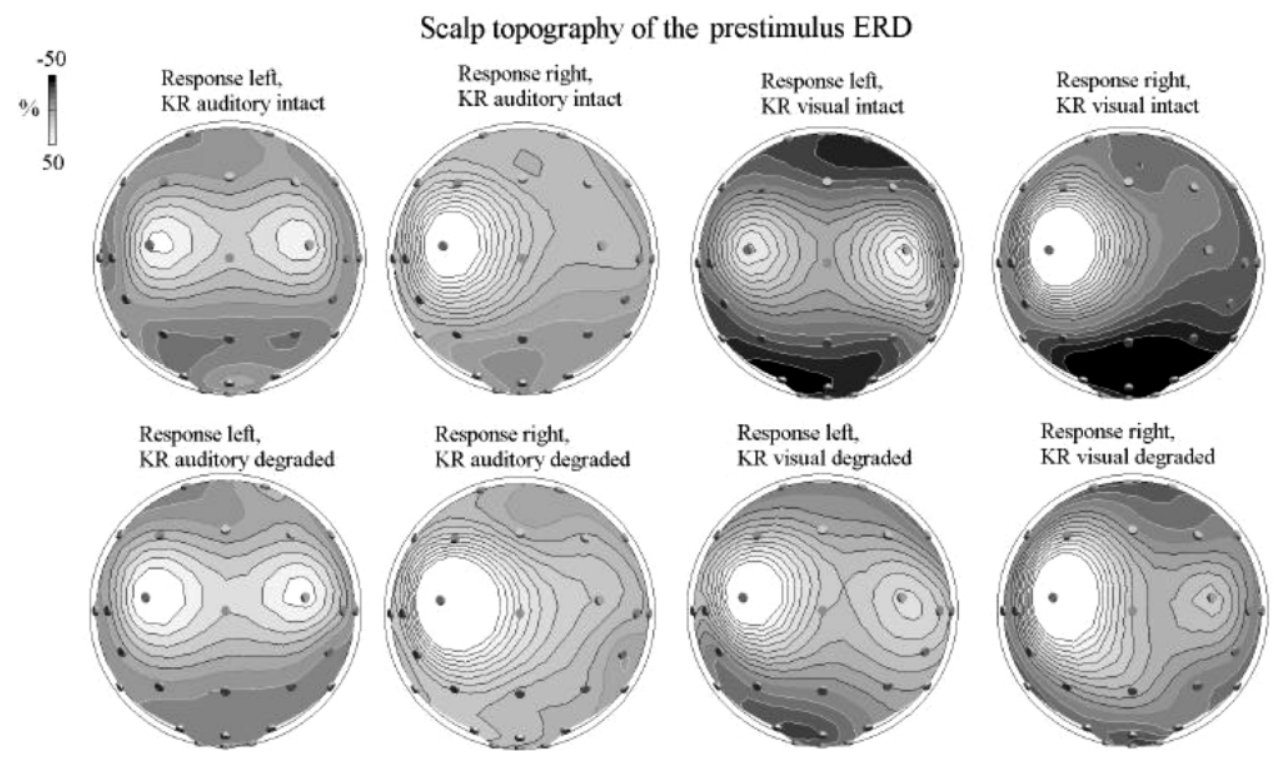

Fig. 5. Topographic maps of the grand average $(N=12)$ ERD in the $8-10-\mathrm{Hz}$ frequency band in the last $250-\mathrm{ms}$ interval preceding the presentation of the KR stimulus. Spacing of isopercentage lines is $5 \%$. Scaling as indicated in the figure. 
experiment, that stimulus degradation results in a smaller ERD (although still significantly different from zero), does indicate a sensitivity of the anticipatory ERD to stimulus parameters, the direction of the effect is opposite to what we expected. It is unclear at present how to interpret this effect.

However, mainly the results of experiments 1-3 at least partially support the notion that anticipatory attention in the auditory and visual modalities is accompanied by an ERD over the temporal and occipital cortices, respectively. Of the remaining three modalities, only the somatosensory modality is easily suitable for applying standardized stimuli; for the olfactory and taste modalities, highly specialized equipment would be needed to standardize stimulus presentation. Therefore, in order to establish a firmer basis for the more general notion that anticipatory attention for an upcoming stimulus manifests itself as an ERD that is restricted to that part of the sensory cortex that corresponds to the modality of the anticipated stimulus, an experiment was performed in which the ERD preceding somatosensory stimuli was investigated. It should be noted that the spatial proximity of the somatosensory and motor cortices may pose a problem for separating these post-movement processes from prestimulus processes. However, for the ERD analyses of the present experiment there are in principle two ways of separating those processes. First, the post-movement ERS has a maximum over the motor cortex contralateral to the movement side. If somatosensory KR stimuli are applied to one location on the body, irrespective of which hand is used for producing the response, the putative pre-stimulus ERD will be independent of response side. Note that such a procedure is probably not very useful for the slow potential data, since the pre-KR SPN typically contains a Response Side * Hemisphere interaction (cf. Damen and Brunia, 1994). Second, the location of the pre-stimulus ERD will correspond to the cortical representation area of the stimulated body part. In the present experiment, somatosensory stimuli will be applied to the subject's leg, therefore the pre-stimulus ERD is expected to be maximal over the post-central midline, whereas post-movement effects are expected to occur at more lateral positions, i.e. over the cortical hand representation areas.

\section{Experiment 4: pre-stimulus ERD in the somatosensory modality}

\subsection{Methods}

In this experiment (described in detail in Bastiaansen, 2000), 13 healthy, right-handed subjects performed a time estimation task, in which visual and somatosensory KR stimuli were used. A voluntary movement task was included as a control condition. The data of two subjects were discarded from further analysis because of the presence of excessive eye movement artifacts, and the data of an additional subject were discarded from further analysis because of the absence of clear $10-\mathrm{Hz}$ peaks in the frequency spectrum of the EEG, which makes ERD analyses meaningless (cf. Pfurtscheller and Lopes da Silva, 1999b). The final subject pool consisted of three males and seven females, aged $18-22(\mathrm{M}=19.8)$. The EEG was recorded from 29 electrodes, and the ERD was computed in the $8-12 \mathrm{~Hz}$ frequency band. The visual KR stimulus consisted of a white vertical bar against a black background, the somatosensory KR stimulus consisted of an electrical pulse applied to the right calf muscle (M. gastrocnemius medialis). The information conveyed by the KR stimuli was encoded by the number of presentations ( $30 \mathrm{~ms}$ on, $120 \mathrm{~ms}$ off) of the visual or somatosensory stimulus. This could be 1,2 , or 4 times, corresponding to an estimated interval that was too short, correct, or too long, respectively.

\subsection{Results}

The main results of the experiment are summarized in Fig. 6. As far as the visual KR conditions are concerned, the results again show an occipital ERD prior to the visual KR stimulus in both the $8-10-\mathrm{Hz}$ and the $10-12-\mathrm{Hz}$ band. Furthermore, as in experiment 3, the post-movement ERS at the pre-KR time interval is larger in the time 


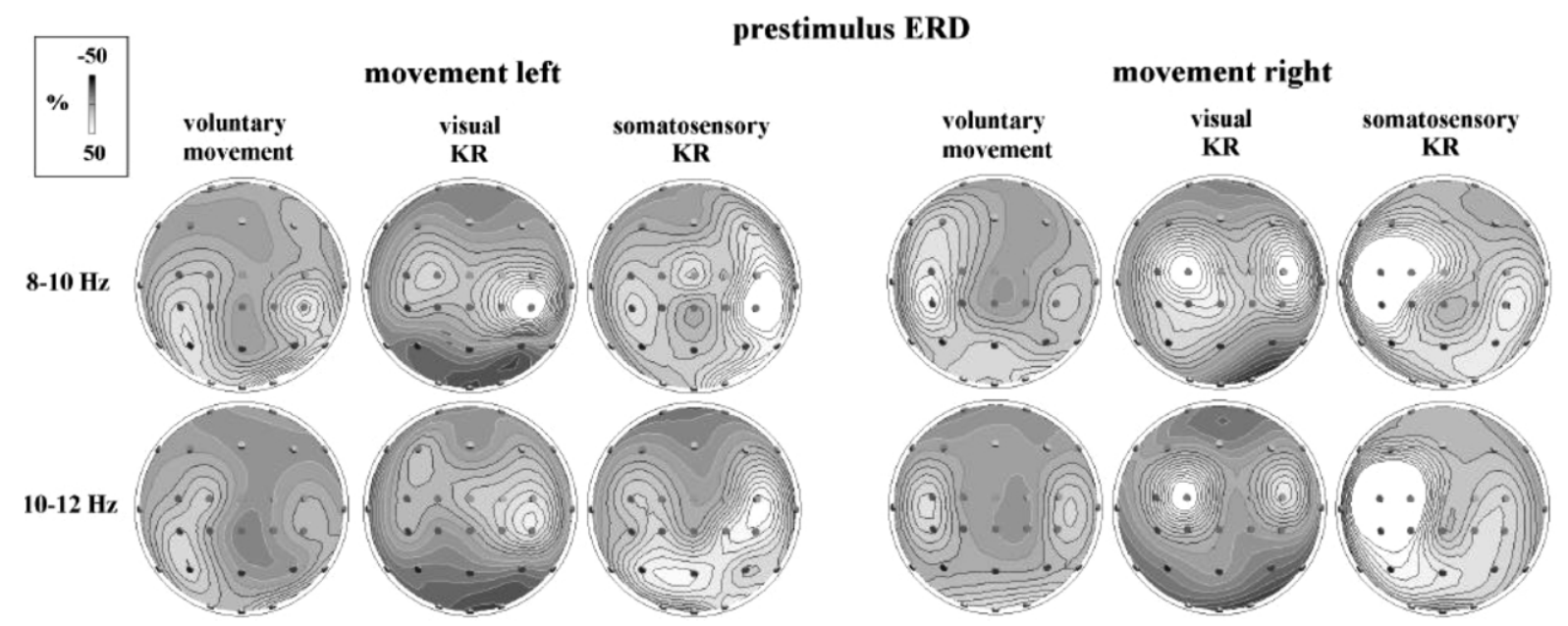

Fig. 6. Grand average $(N=10)$ topographic ERD maps at the pre-KR interval in all three conditions and both frequency bands. Spacing of isopercentage lines is $5 \%$. Scaling as indicated in the figure.

estimation conditions than in the voluntary movement condition. Therefore, a subtraction as in experiment 1 of the voluntary movement conditions from the time estimation conditions cannot be performed with the present data.

In the somatosensory KR condition, the postmovement ERS at the post-central midline is significantly smaller than the post-movement ERS at lateral post-central channels. This effect is present both in the $8-10-\mathrm{Hz}$ and in the $10-12-\mathrm{Hz}$ bands. Interestingly, this reduction in post-movement ERS, from lateral post-central leads to the midline post-central lead, is exclusively present preceding somatosensory KR stimuli, not in the visual KR condition, nor in the voluntary movement condition.

As can be seen in Fig. 6, in the voluntary movement condition an ERD can be identified at the post-central midline in the interval of 1750-2000 ms post-movement (this corresponds with the pre-stimulus interval in the time estimation tasks). However, this ERD does not reach significance (nor does it differ significantly from the ERS present at lateral post-central electrodes, as mentioned above).

\subsection{Interim discussion}

Preceding visual KR stimuli, an occipital ERD was found, that is present neither in the voluntary movement condition nor in the somatosensory $\mathrm{KR}$ condition. This is in agreement with the previous experiments. However, preceding somatosensory KR stimuli the results are less straightforward. In this condition, there is a postmovement ERS at lateral central electrode positions. However, at the post-central midline there is a significant reduction in the post-movement ERS that is not present in the other two conditions. It may be hypothesized that this reduction in ERS is in fact an ERD that is superimposed on the (relatively strong) post-movement ERS.

There are two arguments in favor of such an interpretation. First, the reduction in ERS is localized to the post-central midline, which corresponds to the representation of the leg in the somatosensory cortex. Second, the reduction in ERS is present irrespective of response side. As pointed out above, an effect that is caused by post-movement processes is usually characterized by an interaction with response side and hemisphere, as in the case of the post-movement ERS at lateral post-central electrodes. Therefore, the reduction in ERS at the post-central midline cannot be attributed to post-movement processes. It should be noted that in studies with dense electrode arrays over the sensorimotor cortex, the post-movement ERS is usually restricted to the 
cortical representation area of the moving body part (e.g. Pfurtscheller and Lopes da Silva, 1999b). In contrast, in the present series of experiments, the post-movement ERS is consistently more widespread, extending also to post-central midline electrodes. This is most probably due to the relatively low spatial sampling used in the studies reported here, relative to studies in which large numbers of electrodes are placed over the central areas. The effect of spatial sampling is probably even magnified by the use of spatial derivative methods as Hjorth or spline-based SCDs common in movement-related ERD research, which act as spatial high-pass filters.

In conclusion, the results of experiment 4 suggest that anticipating a somatosensory stimulus is accompanied by a post-central ERD, although because of the paradigm that has been used in this experiment, the post-central ERD is hidden in a post-movement ERS. With respect to the latter, it should be added that the temporal overlap between post-movement processes and prestimulus processes on the one hand, and the spatial proximity of the somatosensory and motor cortices, combined with a relatively low spatial sampling on the other hand, hinders the straightforward identification of the post-central ERD preceding somatosensory stimuli as an independent component.

\section{General discussion}

Taken together, the results of the different experiments show one robust effect, that has been found in all the subjects of all the experiments: this effect is the occipital ERD preceding visual KR stimuli. Therefore, it can be concluded that anticipatory attention for a visual stimulus is accompanied by a pre-stimulus desynchronization of the visual cortex, which we interpret as a reflection of enhanced thalamocortical transfer of sensory information from the thalamus to the visual cortex. In the auditory modality, there is no bilateral temporal pre-stimulus ERD in the EEG data; this finding has been replicated in all three EEG experiments that used auditory KR stimuli. However, in the MEG, such a bilateral temporal pre-stimulus ERD was found in two out of the five subjects studied, which fits well into the results of other research groups who found that rhythmic activity originating from the temporal cortex can be measured with MEG, but not with scalp recorded EEG. In sum, the data only partly support the notion that anticipatory attention for an auditory stimulus is accompanied by an activation of the auditory cortex. In the only experiment in which the somatosensory modality was used, it has been difficult to separate movementrelated processes from pre-stimulus processes. However, the data suggest that anticipatory attention for a somatosensory stimulus is accompanied by an activation of the somatosensory cortex.

A possible explanation for the fact that the clearest results were obtained in the visual modality may be that rhythmic $10-\mathrm{Hz}$ activity originating from the visual cortex (i.e. the alpha rhythm) dominates the EEG/MEG of most individuals, whereas the $10-\mathrm{Hz}$ rhythms from the auditory and somatosensory cortices (the tau and mu rhythms, respectively) are much less prominent. This is probably due to the fact that the visual cortex covers a larger part of the scalp than the somatosensory or auditory cortices. Therefore, the effects in these modalities may be less sizeable than in the visual modality, and as a result the detectability of these effects at the scalp may be more dependent, as compared to the effects in the visual modality, on factors that were not explicitly controlled for. Such factors may be, for example: the signal-to-noise ratio of the measurements; the general level of alertness of subjects; the exact orientation of the sensory cortices relative to the EEG or MEG sensors, etc. Nevertheless, taken over experiments, the general pattern of the results does point to a modality specificity of the pre-stimulus ERD. Therefore, the general research question, 'is anticipatory attention for an upcoming stimulus realized at the neurophysiological level as a pre-stimulus desynchronization of the relevant sensory cortex, reflecting enhanced thalamocortical transfer in the relevant sensory modality?', can be answered positively.

The rationale behind using the time estimation + KR paradigm was to separate movementrelated processes from pre-stimulus processes (cf. 
Damen and Brunia, 1987). Although this strategy has proved to be successful for slow potential research (e.g. Damen and Brunia, 1994), in the case of ERD/ERS analyses, the long-lasting ERS observed after movement hindered an easy separation between the post-movement and the prestimulus effects. By including a voluntary movement condition in most of the experiments (except in experiment 2), it was hoped that movement-related ERD/ERS could be successfully eliminated from the pre-stimulus ERD by a subtraction procedure. Although this worked well in experiment 1 , experiments 3 and 4 showed clear differences in post-movement ERS between the voluntary movement conditions and the time estimation conditions, the post-movement ERS being much stronger in the latter conditions. It might be suggested that the larger post-movement ERS found in the KR conditions is related to 'emitted alpha activity'. Basar and co-workers (e.g. Maltseva et al., 2001) described this phenomenon in an omitted stimulus paradigm. Alpha activity preceding the omitted stimulus showed an anticipatory phase-locking over trials that increased towards the end of the experiment (that is, when anticipation of the omitted stimulus was more prominent as the subject was better trained on the task). This was termed emitted alpha. The increased phase-locking leads to a larger alpha amplitude in the averaged ERP. However, since the increased phase-locking was accompanied by an amplitude attenuation, and ERD/ERS measures are not influenced by the phase, but exclusively by the amplitude of a signal, one would rather expect an ERD rather than an ERS to result from the phenomenon of emitted alpha. Therefore, the larger post-movement ERS in the KR conditions cannot be explained by the phenomenon of emitted alpha. Since it has repeatedly been shown that post-movement ERS is strongly influenced by movement parameters (e.g. Pfurtscheller et al., 1999; Stancak and Pfurtscheller, 1999), an alternative explanation of the results might be that the time estimation task imposed higher constraints on certain movement parameters (e.g. the timing of the movement) than the voluntary movement task, therewith leading to a larger post-movement ERS in the former task.

Klimesch et al. $(1992,1998)$ have suggested that the lower alpha band (i.e. $8-10 \mathrm{~Hz}$ ) might be more sensitive to anticipatory processes than the upper alpha band. The results described in the present paper do not support this notion, however. A possible reason for the discrepancy between the Klimesch et al. results and the present results may be that in the present paper, fixed frequency bands may be used, as opposed to the procedure of individual frequency band selection used by Klimesch et al. The fixed-band approach may have blurred differences between upper and lower alpha in the present analyses.

Finally, it is interesting to note that in the data presented in this paper, a clear functional dissociation is present between the pre-stimulus ERP (the SPN) and the pre-stimulus ERD. Elsewhere (Bastiaansen et al., 2001b), it has been argued that in general SPN reflects the presetting of cortical areas that are involved in the execution of the task at hand. In the time estimation paradigm that has been used in this research, the pre-KR SPN most probably reflects the anticipation of the affective-motivational properties of the KR stimulus. On the other hand, the prestimulus ERD that has been computed on the same data most likely reflects perceptual anticipatory attention, as has been argued above. The important implication of the functional dissociation between ERPs and ERD, that was found in the experiments reported here, is that different neurophysiological processes can be captured by the different analysis methods. The data presented in the present paper are globally in line with the notion that studying patterns of ERD/ERS in the alpha frequency range may specifically provide us with a window on the processes operating in thalamocortical circuits (Steriade et al., 1990; Lopes da Silva, 1991; Guillery et al., 1998). It should be noted here that ERD/ERS in other frequency bands most probably reflects different neurophysiological mechanisms; for instance, it has been proposed that ERD/ERS in the theta frequency range is a reflection of cortico-hippocampal interactions (e.g. 
Miller, 1991), a proposition that is supported by a clear relationship between memory performance and event-related theta responses (see Klimesch, 1999, for a discussion and a literature review). An implication of the functional dissociation between slow potentials and ERD that was found in this research is that it may be advantageous in some cases to use both ERD and ERP measures in the analysis of EEG/MEG recordings.

\section{Acknowledgements}

The research described in this paper was funded by the Cooperation Centre Tilburg and Eindhoven Universities (SOBU), grant 1995 AD. At the time of manuscript preparation, the author was funded by grant 400-56-384 of the Dutch Organization for Scientific Research (NWO). Thanks to Gert Pfurtscheller, Christa Neuper and Koen Böcker for valuable comments on an earlier version of the manuscript.

\section{References}

Bastiaansen, M.C.M., 2000. Anticipatory attention: an EventRelated Desynchronization approach. Ph.D thesis, Tilburg University.

Bastiaansen, M.C.M., Knösche, T.R., 2000. MEG tangential derivative mapping applied to Event-Related Desynchronization (ERD) research. Clin. Neurophysiol. 111, $1300-1305$.

Bastiaansen, M.C.M., Brunia, C.H.M., Böcker, K.B.E., 1999a. ERD as an index of anticipatory behavior. In: Pfurtscheller, G., Lopez da Silva, F. (Eds.), Event-Related Desynchronization. Handbook of Electroencephalography and Clinical Neurophysiology (revised series), 6. Elsevier, Amsterdam, pp. 203-217.

Bastiaansen, M.C.M., Böcker, K.B.E., Cluitmans, P.J.M., Brunia, C.H.M., 1999b. Event-Related Desynchronization related to the anticipation of a stimulus providing Knowledge of Results. Clin. Neurophysiol. 110, 250-260.

Bastiaansen, M.C.M., Böcker, K.B.E., Brunia, C.H.M., De Munck, J.C., Spekreijse, H., 2001a. Event-Related Desynchronization during anticipatory attention for an upcoming stimulus: a comparative EEG/MEG study. Clin. Neurophysiol. 112, 393-403.

Bastiaansen, M.C.M., Böcker, K.B.E., Brunia, C.H.M, 2001b. ERD as an index of anticipatory attention? Effects of stimulus degradation. Psychophysiology, submitted for publication.
Böcker, K.B.E., Brunia, C.H.M., Van den Berg-Lenssen, M.M.C., 1994. A spatiotemporal dipole model of the stimulus-preceding negativity (SPN) prior to feedback stimuli. Brain Topogr. 7, 71-88.

Böcker, K.B.E., Van Boxtel, G.J.M., 1997. Stimulus-preceding negativity: a class of anticipatory slow potentials. In: Van Boxtel, G.J.M., Böcker, K.B.E. (Eds.), Brain and Behavior: Past, Present and Future. Tilburg University Press, Tilburg, pp. 105-116.

Brunia, C.H.M., 1999. Neural aspects of anticipatory behavior. Acta Psychol. 101, 213-242.

Brunia, C.H.M., De Jong, B.M., Van Den Berg-Lenssen, M.M.C., Paans, A.M.J., 2000. Visual feedback about time estimation is related to a right-hemisphere activation measured by PET. Exp. Brain Res. 130, 328-337.

Bullock, T.H., 1992. Introduction to induced rhythms: a widespread, heterogeneous class of oscillations. In: Basar, E., Bullock, T.H. (Eds.), Induced Rhythms in the Brain. Birkhauser, Boston, pp. 1-26.

Damen, E.J.P., Brunia, C.H.M., 1987. Changes in heart rate and slow brain potentials related to motor preparation and stimulus anticipation in a time estimation task. Psychophysiology 24, 700-713.

Damen, E.J.P., Brunia, C.H.M., 1994. Is a stimulus conveying task-relevant information a sufficient condition to elicit a stimulus-preceding negativity? Psychophysiology 31, 129-139.

Guillery, R.W., Feig, S.L., Loszadi, D.A., 1998. Paying attention to the thalamic reticular nucleus. Trends Neurosci. 21, $28-32$.

Hjorth, B., 1975. An on-line transformation of EEG scalp potentials into orthogonal source derivations. Electroencephalogr. Clin. Neurophysiol. 39, 526-530.

Klimesch, W., 1999. EEG alpha and theta oscillations reflect cognitive and memory performance: a review and analysis. Brain Res. Rev. 29, 169-195.

Klimesch, W., Pfurtscheller, G., Schimke, H., 1992. Pre- and poststimulus processes in category judgment tasks as measured by Event-Related Desynchronization (ERD). J. Psychophysiol. 6, 186-203.

Klimesch, W., Doppelmayr, M., Russegger, H., Pachinger, Th., Schweiger, J., 1998. Induced alpha band power changes in the human EEG and attention. Neurosci. Lett. 244, 73-76.

Lopes da Silva, F.H., 1991. Neural mechanisms underlying brain waves: from neural membranes to networks. Electroencephalogr. Clin. Neurophysiol. 79, 81-93.

Lehtelä, L., Salmelin, R., Hari, R., 1997. Evidence for reactive magnetic $10-\mathrm{Hz}$ rhythm in the human auditory cortex. Neurosci. Lett. 222, 111-114.

Miller, R., 1991. Cortico-hippocampal Interplay and the Representation of Contexts in the Brain. Springer, Berlin.

Niedermeyer, N., 1990. Alpha-like rhythmical activity of the temporal lobe. Clin. Electroencephalogr. 21, 210-224.

Niedermeyer, E., 1991. The 'third rhythm': further observations. Clin. Electroencephalogr. 22, 83-96. 
Perrin, F., Bertrand, O., Pernier, J., 1987. Scalp current density mapping: value and estimation from potential data. IEEE Trans. Biomed. Eng. 34, 283-288.

Perrin, F., Pernier, J., Bertrand, O., Echallier, J.F., 1989. Spherical splines for scalp potential and current density mapping. Electroencephalogr. Clin. Neurophysiol. 72, 184-187.

Pfurtscheller, G., 1991. Mapping procedures. In: Weitkunat, R. (Ed.), Digital Biosignal Processing. Elsevier, Amsterdam, pp. 459-480.

Pfurtscheller, G., 1999. Quantification of ERD and ERS in the time domain. In: Pfurtscheller, G., Lopes da Silva, F.H. (Eds.), Event-Related Desynchronization. Handbook of Electroencephalography and Clinical Neurophysiology (revised series), 6. Elsevier, Amsterdam, pp. 89-105.

Pfurtscheller, G., Lopes da Silva, F.H., 1999a. Elsevier, Amsterdam 405 pp..

Pfurtscheller, G., Lopes da Silva, F.H., 1999b. Event-related EEG-MEG synchronization and desynchronization: basic principles. Clin. Neurophysiol. 110, 1842-1857.

Pfurtscheller, G., Pichler-Zalaudek, K., Neuper, C., 1999. ERD and ERS in voluntary movement of different limbs. In: Pfurtscheller, G., Lopez da Silva, F. (Eds.), Event-Related Desynchronization. Handbook of Electroencephalography and Clinical Neurophysiology (revised series), 6. Elsevier, Amsterdam, pp. 245-268.

Requin, J., Brener, J., Ring, C., 1991. Preparation for action. In: Jennings, J.R., Coles, M.G.H. (Eds.), Handbook of Cognitive Psychophysiology: Central and Autonomic Nervous System Approaches. Wiley, Chisester, pp. 357-448.

Rockstroh, B., Müller, M., Heinz, A., Wagner, M., Berg, P., Elbert, T., 1996. Modulation of auditory responses during oddball tasks. Biol. Psychol. 43, 41-55.

Stancak, A., Jr., Pfurtscheller, G., 1999. Effects of handedness and type of movement on Event-Related Desynchronization within the alpha and beta bands. In: Pfurtscheller, G., Lopez da Silva, F. (Eds.), Event-Related Desynchronization. Handbook of Electroencephalography and Clinical Neurophysiology (revised series), 6. Elsevier, Amsterdam, pp. 269-278.

Steriade, M., Gloor, P., Llinás, R.R., Lopes da Silva, F.H., Mesulam, M.M., 1990. Basic mechanisms of cerebral rhythmic activities. Electroencephalogr. Clin. Neurophysiol. 76, 481-508.

Tiihonen, J., Hari, R., Kajola, M., Karhu, J., Ahlfors, S., Tissari, S., 1991. Magnetoencephalographic $10 \mathrm{~Hz}$ rhythm from the human auditory cortex. Neurosci. Lett. 129, 303-305. 